\title{
LES NODULES POLYMÉTALLIQUES ET LA DÉPOLLUTION DES EAUX
}

\section{Polymetallic nodules and water depollution}

\author{
G. PIGNET \\ G. MARTIN \\ (EEA. ENSC: Rennes \\ Avenue du Ciénéral-Leclerc \\ 35000 Rennes-Beaulieu
}

H. BOUGAULT

COB - CINEXO

B. P. 337

29273 Brest

\section{INTRODUCTION}

Les eaux superficielles sont atteintes de plus en plus gravement par la pollution industrielle, alors que les besoins en eau pure augmentent. C'est pourquoi on est amené à utiliser pour la consommation des eaux de qualités variables contenant en particulier un certain nombre de micropolluants. Les essais présentés ici ont pour but de tester une méthode originale d'élimination de différents cations métalliques bivalents.-

$$
\mathrm{Mn}^{2+}, \mathrm{Cu}^{2+}, \mathrm{Zn}^{2+}
$$

Le manganèse pose un problème particulièrement important puisqu'il peut atteindre des teneurs de 0,5 à $1 \mathrm{mg} / \mathrm{l}$ dans les eaux de barrage.
Le traitement le plus courant consiste à oxyder $\mathrm{Mn}^{2+}$ en $\mathrm{MnO}_{2}$ insoluble que l'on filtre. Ce traitement qui nécessite l'emploi d'un oxydant puissant tel que le permanganate de potassium est relativement coûteux.

Pour les autres métaux présents éventuellement dans les caux de ruissellement par exemple, il n'existe pas pour l'instant de traitement spécifique applicable aux eaux de consommation. I.es teneurs de ces métaux dans les eaux de barrage ou eaux de ruissellement, les normes de potabilité et l'efficacité de certains traitements sont donnés tableau I. 
TABLEAU I

Les métaux dans les eaux superficielles, normes de potabilité et efficacité de certains traitements

\begin{tabular}{|c|c|c|c|c|c|c|}
\hline & $\mathrm{Mn}$ & $\mathrm{Cu}$ & $\mathrm{Ni}$ & $Z \mathrm{n}$ & $\mathrm{Pb}$ & $\mathrm{Hg}$ \\
\hline $\begin{array}{ll}\text { Teneur } & \text { eaux à caractère } \\
\text { max. } & \text { particulier } \\
\text { mg/l } & \text { eaux de rivière }\end{array}$ & $\begin{array}{c}\text { eaux de barrage } \\
2 \\
0,06\end{array}$ & \multicolumn{4}{|c|}{ eaux de ruissellement } & \\
\hline $\begin{array}{l}\text { Normes de potabilité } \mathrm{mg} / 1 \\
\text { (comm. Europ. 1976) }\end{array}$ & 0,05 & 3 & 0,05 & 5 & 0,05 & 0,001 \\
\hline $\begin{array}{l}\text { Traitements spécifiques } \\
\text { teneurs résicluelles mg/l }\end{array}$ & $\begin{array}{l}\text { oxydation } \\
\text { filtration } \\
<0,01\end{array}$ & \multicolumn{3}{|c|}{$\begin{array}{l}\text { précipitation à } \\
\text { l'hydroxyde }\end{array}$} & \multicolumn{2}{|c|}{$\begin{array}{l}\text { précipitation } \\
\text { du sulfure }\end{array}$} \\
\hline $\begin{array}{l}\text { Traitement classique } \\
\text { (coag., flocul. décant. filt.) } \\
\text { \% d'abattement pour des } \\
\text { teneurs d'entrée de } 0,5 \mathrm{mg} / 1\end{array}$ & $20 \%$ & $\begin{array}{c}80 \\
\dot{a} \\
90 \%\end{array}$ & $\begin{array}{c}20 \\
\text { à } \\
65 \%\end{array}$ & $\begin{array}{c}30 \\
\text { à } \\
40 \%\end{array}$ & $\begin{array}{c}60 \\
\grave{a} \\
80 \%\end{array}$ & $70 \%$ \\
\hline
\end{tabular}

Les nodules polymétalliques sont des concrétions naturelles que l'on trouve dans les grands fonds océaniques par des profondcurs comprises en général entre 3500 et 5000 mètres. Les deux ćléments majeurs sont le fer et le manganèse, cn première approximation sous forme d'oxydes $\mathrm{Fe}_{2} \mathrm{O}_{3}$ et $\mathrm{MnO}_{2}$ : les structures cristallines (birnesite, todorokite) sont très difficiles à mettre en évidence. Nickcl, cuivre et cobalt accompagnent ces deux éléments dans des proportions variables (de l'ordre de grandeur de $1 \%$ ) suivant les zones géographiques. Le tableau II donne la composition d'un nodule "moyen" ainsi que les concentrations en $\mathrm{Mn}, \mathrm{Fe}, \mathrm{Ni}$ et $\mathrm{Cu}$ des nodules «Walda $\left(^{*}\right)$ utilisés lors de cette étude. La source des ćléments qui les constituent (volcanisme, phénomènes hydrothermaux, apports continentaux...) leurs processus et vitesse de formation ne sont pas actuellement connus avec certitude. Dans certaines zones géographiques, notamment dans le Pacifique Nord, ces nodules polymétalliques présentent des teneurs cumulées « $\mathrm{Ni}$ + $\mathrm{Cu}$ " supérieures à $3 \%$ qui en font un minerai potentiel (GLASBy, 1977). Les travaux décrits dans cet article envisagent une possibilité

(*) "Walda": mission N.O. J. Charcot en 1971. Ces nodules ont été prélevés dans le bassin d'Angola, en bas de marge. d'application de ce type de matériau au traitement des eaux (Pignet, 1978).

La possibilité d'utilisation des nodules polymétalliques pour le traitement des eaux repose sur deux observations :

i) Les nodules polymétalliques dont les dimensions varient du centimètre à la dizaine de centimètres sont formés de couches successives d'oxydes de manganèse (et de fer). On sait que le dioxyde de manganèse possède des propriétés catalytiques vis-à-vis de ccrtaines réactions d'oxydation.

ii) Le matériau possède unc structure porcuse très divisée favorable aux réactions de surface.

Ces deux remarques ont conduit à envisager l'emploi des nodules de manganèse comme absorbants et catalyseurs d'oxydation. Les chances de pouvoir "fixer» certains éléments sur ce matériau apparaissent a priori d'autant plus grandes que ces éléments peuvent intervenir dans la composition et la formation du matériau naturel. On espère donc que le processus naturcl qui intervient pour la formation du matériau peut être reproduit en laboratoire ou en station d'épuration autorisant ainsi l'élimination d'ions tels que $\mathrm{Mn}^{2+}, \mathrm{Cu}^{2+}, \mathrm{Zn}^{2+} \ldots$ contenus dans certaines eaux.

TABLEAAU II

Composition des nodules Walda

Comparaison à la composition moyenne des nodules.

\begin{tabular}{|c|ccccccccccc|}
\hline $\begin{array}{c}\text { Nodule } \\
\text { en } \\
\text { "moyen }\end{array}$ & $\mathrm{Mn}$ & $\mathrm{Fe}$ & $\mathrm{Si}$ & $\mathrm{Al}$ & $\mathrm{Na}$ & $\mathrm{Ca}$ & $\mathrm{Mg}$ & $\mathrm{Ni}$ & $\mathrm{K}$ & $\mathrm{Ti}$ & $\mathrm{Cu}$ \\
\hline $\begin{array}{c}\text { Nodule } \\
\text { "Walda }\end{array}$ & $\mathbf{2 4 , 2}$ & $\mathbf{1 4}$ & $\mathbf{9 , 4}$ & 2,9 & 2,6 & 1,9 & 1,7 & 0,99 & 0,8 & 0,67 & 0,5 \\
\hline 40 & 0,9 & & & & & & .11 & & & & \\
\hline What
\end{tabular}




\section{ÉTUDE DE L'ÉLIMINATION DU MANGANÈSE}

\section{Conditionnement}

Le conditionnement des nodules doit permettre de profiter au maximum de leurs propriétés de surface : il est donc nécessaire de les concasser. La granulométrie a été définie pour cette étude de 1 à $2,5 \mathrm{~mm}$.

L'aire spécifique est alors de environ $50 \mathrm{~m}^{2} / \mathrm{g}$, ce qui reste très inférieur à celle des produits spécifiquement absorbants tels que le charbon actif de même granulométrie. La densité voisine de 1,1 est faible par rapport à celle du sable. Le conditionnement est complété par un lavage à l'eau à ébullition destiné à " mouiller » au maximum le matériau et à enlever diverses impuretés.

\section{Essais préalables en discontinu}

Les essais préalables en discontinu se sont révélés très encourageants. Dans un réacteur agité mécaniquement, on introduit successivement les nodules préparés $\left(40 \mathrm{~cm}^{3}\right)$ et la solution à traiter (environ 1 litre). Cette solution est préparée à partir d'eau permutée dans laquelle on dissout un sel du métal étudié de façon à obtenir la concentration initiale souhaitée. Chaque expérience consiste à suivre la diminution avec le temps de la concentration en cation.

La première expérience permet de comparer l'efficacité des nodules à celle de matériaux traditionnels tels que pyrolusite et sable manganisé pour l'élimination du manganèse. Cette expérience dont les résultats sont représentés figure 1 montre sans ambiguïté la supériorité des nodules aussi bien vis-à-vis de la concentration en $\mathrm{Mn}$ résiduelle dans l'eau que de la rapidité de l'épuration. Notons que la granulométrie du sable manganisé utilisé est la même que celle du matériau "nodule " concassé.

Ce résultat important étant acquis, la cinétique de la réaction a été étudiée : influence de l'agitation, de la quantité de nodule utilisée, $\mathrm{du} \mathrm{pH}$ et de l'oxygène dissout.

\section{Influence de l'agitation et de la quantité de nodules utilisée}

Les résultats concernant l'influence de l'agitation sont traduits figure 2 , où le logarithme de la concentration en $\mathrm{Mn}^{2+}$ en solution est porté en fonction du temps. En l'absence d'agi-

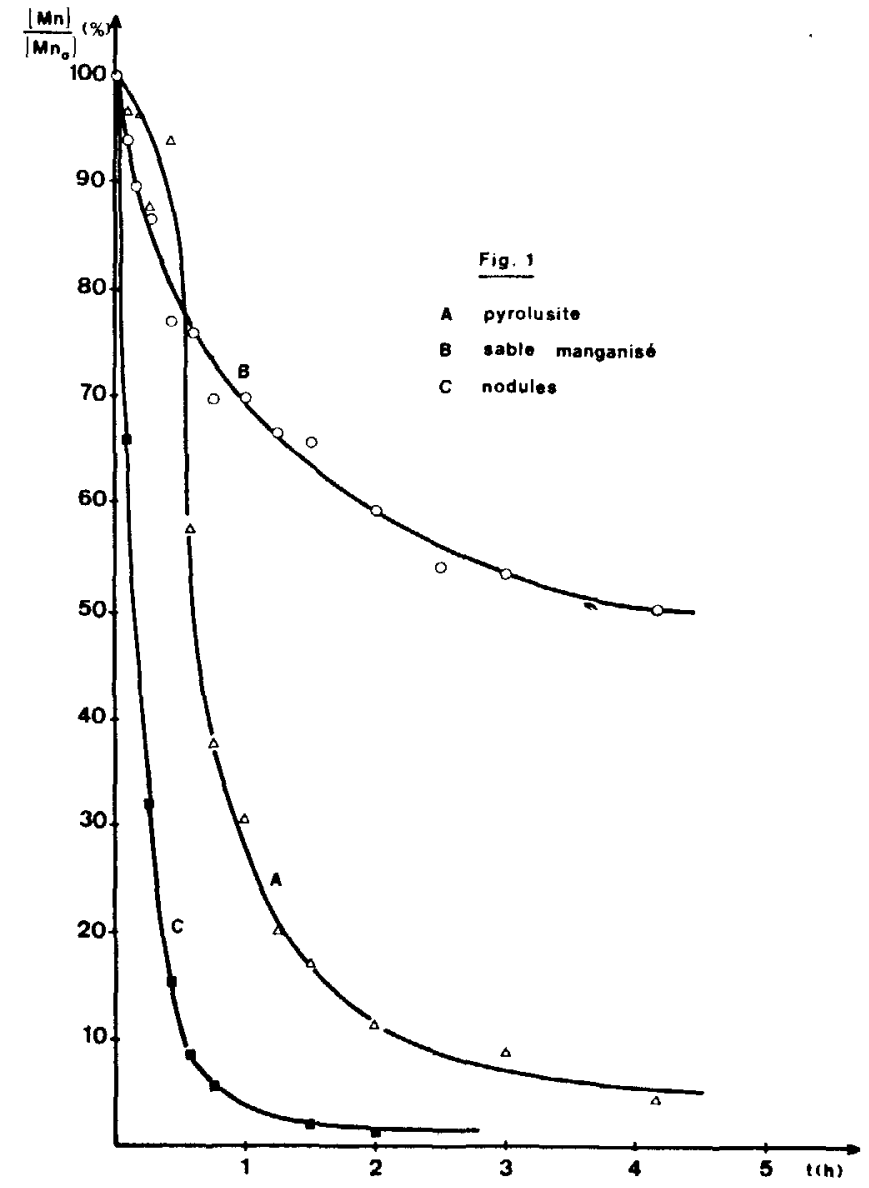

Figure 1

Fifficacté comparée de la pyrolusite, du sable manganisé et des nodules.

$$
\begin{aligned}
& \text { Compared efficiency of pyrolusite, } \\
& M n \text { coated sans and nodules. }
\end{aligned}
$$

tation la diminution de $\mathrm{Mn}^{2+}$ en solution est beaucoup moins rapide qu'avec une agitation. Il a été vérifié que ce résultat ne correspond pas à unc absence de diffusion de $\mathrm{Mn}^{2+}$ en solution dans le cas d'absence d'agitation. Un calcul simple permet de montrer que le volume utile de nodule en absence d'agitation est dans le rapport 1,5 à 42 par comparaison à l'expérience avec agitation. Outre l'influence de l'agitation, ce résultat montre le rôle de la quantité de nodule utilisée sur la cinétique de l'élimination du manganèse. Cette influence a d'ailleurs été précisée par une séric de trois expériences - avec agitation - en utilisant des quantités différentes de nodule. 


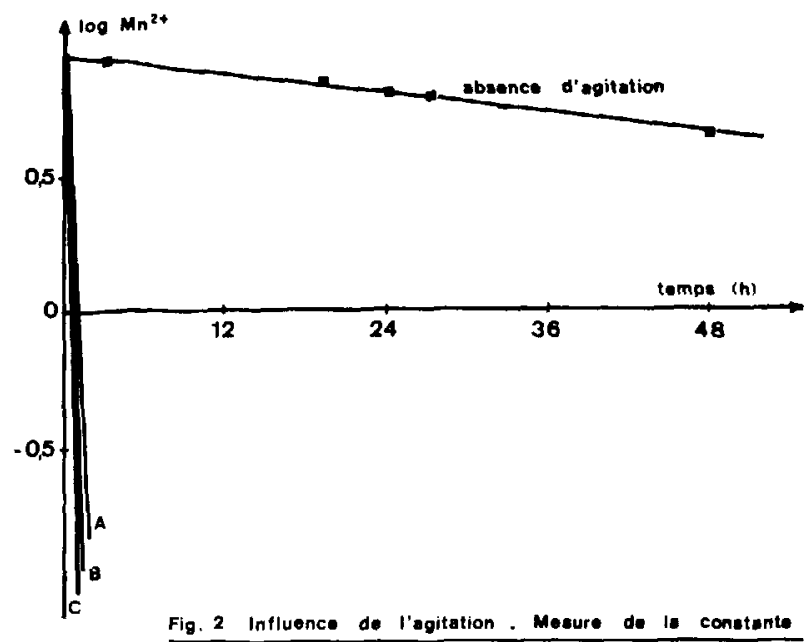

FIGURE 2

Influence de l'agitation.

$\mathrm{A}, \mathrm{B}, \mathrm{C}$ correspondant à trois expériences avec agitation. A : $4 \mathrm{ml}$ de nodule ; $B$ : $20 \mathrm{ml}$; $\mathrm{C}: 40 \mathrm{ml}$ pour 11 d'eau.

Shaking effect; the upper line: no shaking.

$A, B, C$ three experiments with shaking.

$A: 4 \mathrm{ml}$ of nodule; $B: 20 \mathrm{ml} ; C: 40 \mathrm{ml}$ for $1 \mathrm{l}$ of rerater.

En supposant que la réaction suit un ordre n défini,

on écrit $-\frac{\mathrm{dc}}{\mathrm{dt}}=\mathrm{K}_{\mathrm{c}^{\mathrm{n}}}$

dc

soit $\log \left(-\frac{\mathrm{d}}{\mathrm{dt}}\right)=\log \mathrm{K}+\mathrm{n} \log \mathrm{c}$

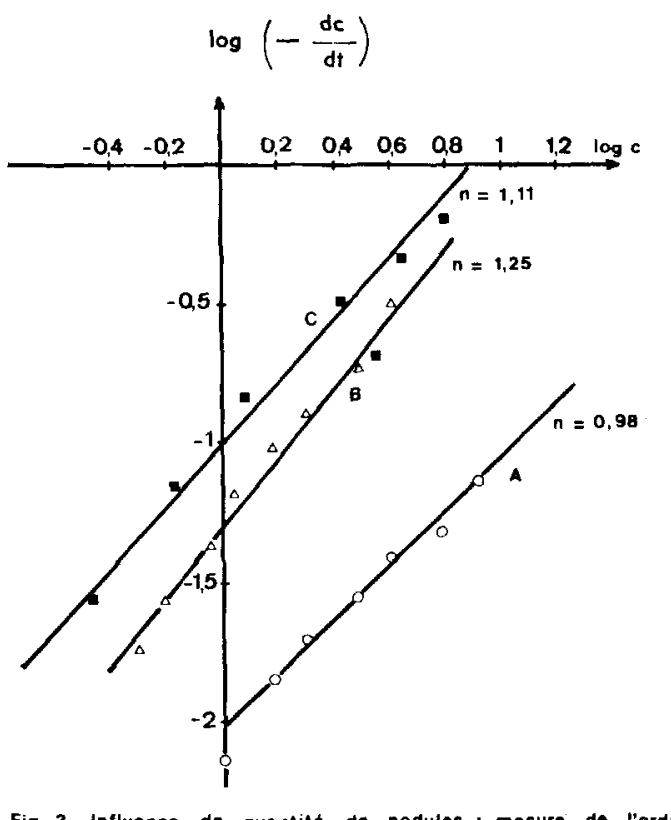

FIGURE 3

Influence de la quantité de nodule.

Ordre de réaction :

A : 4 ml de nodule ; B : $20 \mathrm{ml}$; C : $40 \mathrm{ml}$ pour 11 d'eau.

Influence of nodule quantity : reaction order.

$A: 4 \mathrm{ml}$ of nodule ; $B: 20 \mathrm{ml} ; C: 40 \mathrm{ml}$-water $1 \mathrm{l}$.
Les trois expériences effectuées à quantités de nodules différentes peuvent permettre de préciser $\mathrm{n} ; \log (-\mathrm{dc} / \mathrm{dt})$ en fonction de $\log \mathrm{c}$ est représenté figure 3. L'ordre trouvé est voisin de 1 . Les constantes cinétiques $K$ relatives à chaque expérience peuvent être déterminées en portant $\log c$ en fonction du temps (fig. 4). On retrouve un résultat comparable à ce qui est obtenu lors de l'expérience «absence d'agitation" (fig. 2), où l'absence d'agitation est équivalente à une quantité de nodule réduite.

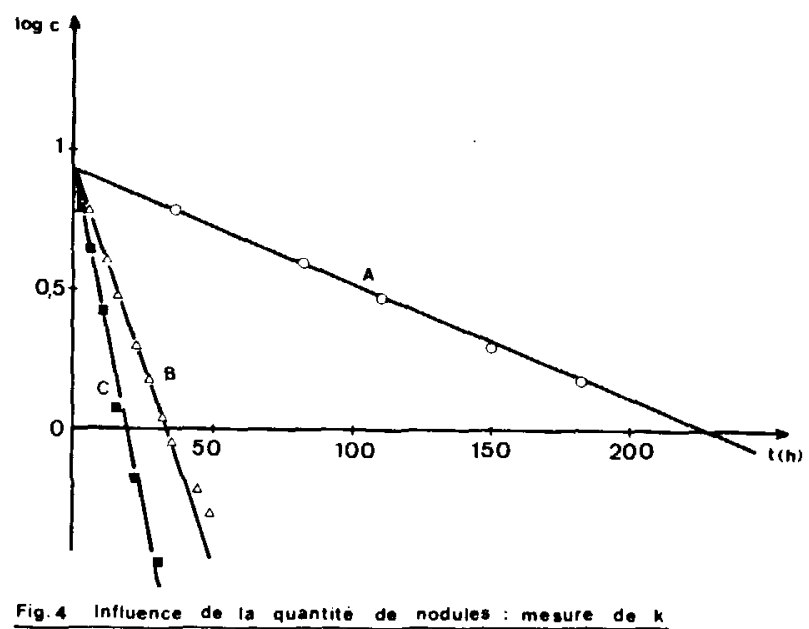

FIGVRE, 4

Influence de la quantité de nodule.

Détermination des constantes cinétiques :

A : $4 \mathrm{ml}$ de nodule ; $\mathrm{B}: 20 \mathrm{ml} ; \mathrm{C}: 40 \mathrm{ml}$ pour 11 d'eau. Kinetic constant determination :

$A: 4 \mathrm{ml}$ of nodule; $B: 20 \mathrm{ml} ; C: 40 \mathrm{ml}$ for $1 \mathrm{l}$ of water.

\section{Influence du $\mathbf{p H}$}

La réaction catalysée ou non d'oxydation de $\mathrm{Mn}^{++}$peut s'écrire (Coughlin et al., 1976)

$\mathrm{Mn}^{++}+3 \mathrm{H}_{2} \mathrm{O}+\frac{\mathrm{x}-1}{2} \mathrm{O}_{2} \rightarrow 2 \mathrm{H}_{3} \mathrm{O}^{+}+\mathrm{MnO}_{\mathrm{x}}$ x pouvant varier de 1,5 à 2 .

Une diminution très significative du $\mathrm{pH}$ est effectivement observéc au cours de la réaction. Trois expériences ont été réalisées pour tester l'influence du $\mathrm{pH}$ sur la cinétique de démanganisation : $\mathrm{A}$ : le $\mathrm{pH}$ évolue librement, variant de 8,3 en début d'expérience à 7,5 en fin d'expérience; $\mathrm{B}: \mathrm{pH}$ maintenu à 8,5 ; $\mathrm{C}: \mathrm{pH}$ maintenu à 5,9 . 
Ces différents $\mathrm{pH}$ n'ont aucune influence sur la cinétique de la réaction comme le montrent les résultats présentés figure 5 . La réaction considérée n'est donc pas réversible.

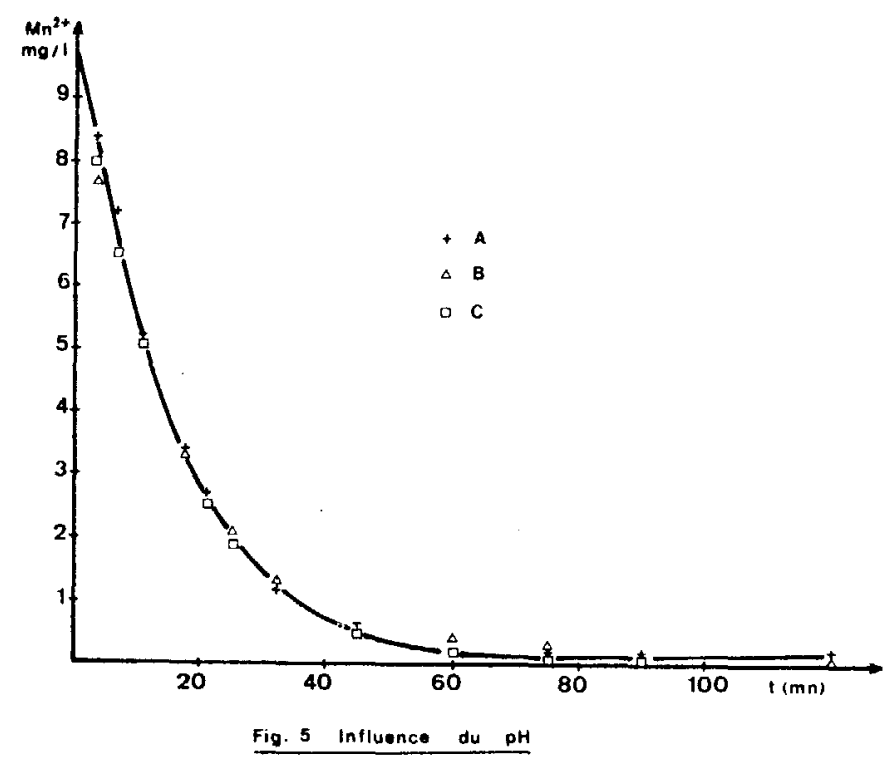

FIGURE 5

Influence négligeable du $\mathrm{pH}$ sur la cinétique. Negligeable influence of $p H$ for kinetics.

\section{Influence de l'oxygène dissout}

La nécessité de présence d'oxygène pour l'élimination du manganèse en solution est démontréc par une expérience en milieu réducteur (sulfite de sodium).

Tant que la solution reste en milieu réducteur, la concentration en manganèse reste constante. Si on fait passer un courant d'oxygène dans la solution, dès que la concentration en $\mathrm{O}_{2}$ devient mesurable la concentration en $\mathrm{Mn}^{2+}$ et le $\mathrm{pH}$ diminuent.

Réaliser la même expérience (absence d' $\mathrm{O}_{2}$ ), sans faire intervenir un agent réducteur, est difficile, le matériau nodule de manganèse pouvant être donneur d'oxygène lui-même ne serait-ce que par l'air emprisonné dans les pores du matériau. En utilisant de l'eau préalablement bouillie, en y maintenant un bullage d'azote le taux d'oxygène peut être maintenu aux environs de $0,05 \mathrm{mg} / \mathrm{l}$. Dans ces conditions, on observe une certaine démanganisation; l'ordre de la réaction est voisin de 2 et la constante cinćtique $\mathrm{K}$ est faible.

\section{"Réactivation " du matériau}

I a démanganisation d'une solution peut s'effectuer en la faisant percoler sur une colonne de nodules. $\mathrm{Au}$ bout d'un certain temps, la colonne se «sature", les grains de nodules perdant leur propriété catalytique. En laissant une solution contenant $\mathrm{Mn}^{2+}$ en contact avec une charge de nodules ainsi saturée, on observe que la concentration en $\mathrm{Mn}^{2+}$ décroît avec le temps : le matériau se "réactive».

Dans ce cas (fig. 6), l'ordre de la réaction est voisin de deux.

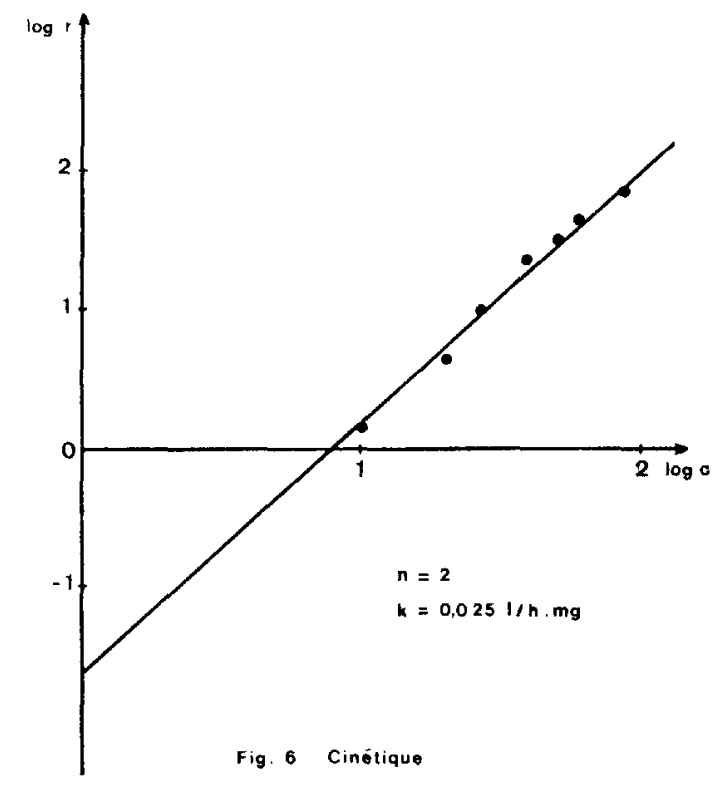

FigURE: 6

"Réactivation du matériau nodule de manganèse.

I e matériau a été préalablement saturé; la pente de droite a $\log \mathrm{r}=\mathrm{f}$ (c) définit l'ordre de la réaction correspondant à la démanganisation d'une solution dont la concentration initiale était de $122 \mathrm{mg} / 1$.

Mangenase nodule reactivation. The material was Freviously saturated; the slope of the line $\log r=f(c)$ is the order of the reaction corresponding to manganese removal from a solution whose initial concentration was $122 \mathrm{mg} / \mathrm{l}$.

\section{Cinétique de la réaction de démanganisation}

Suivant les paramètres et les conditions expérimentales qui ont été étudiées, l'ordre de la réaction est 1 ou 2 ou compris entre 1 et 2. Empiriquement, une relation du type suivant peut être proposée :

$$
-\frac{\mathrm{dc}}{\mathrm{dt}}=\mathrm{Kc}(1+\mathrm{ac})
$$




\section{G. PIGNET, G. MARTIN, H. BOUGAULT}

I.es valeurs des constantes $\mathrm{K}$ et a pouvant varier suivant les conditions de l'expérience. L'ordre de la réaction $n$ est égal à 1 si a est petit; $n$ peut être égal à 2 si (ac) est grand devant 1 et mal défini dans les cas intermédiaires.

Pour un ordre 1 , il a été montré que la vitesse de réaction est proportionnelle à la quantité de nodules utilisée, donc à la surface présentée par le matériau; la constante $K$ peut donc être remplacée par K'S, S étant la surface. Une série d'expéricnces a permis de conclure d'autre part que le paramètre a est inversement proportionnel à $c_{o}^{2}, c_{o}$ étant la concentration initiale en manganèse (fig. 7).

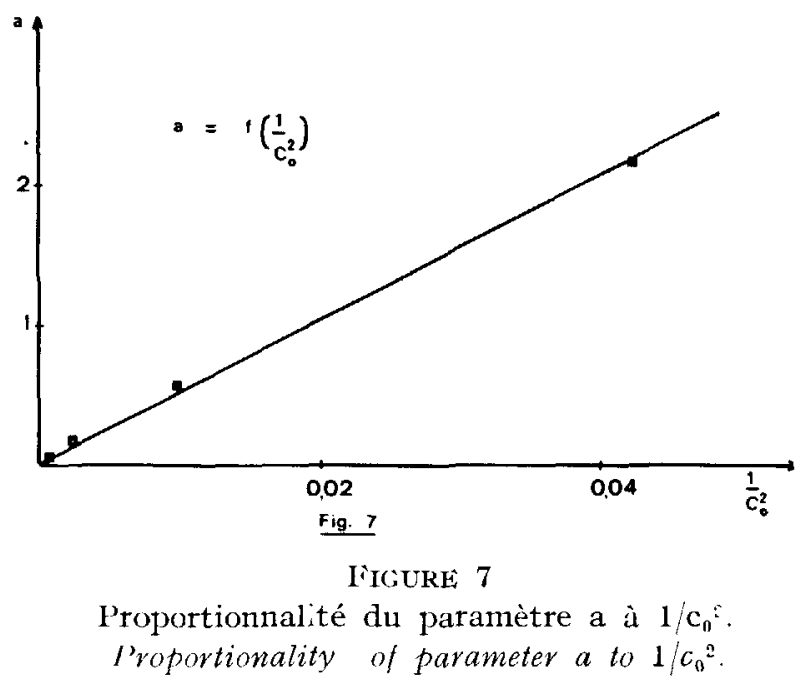

Ainsi, empiriquement la vitesse de démanganisation peut s'exprimer par la relation suivante :

$$
-\frac{\mathrm{dc}}{\mathrm{dt}}-\mathrm{K}^{\prime} \mathrm{Sc}\left(1+\frac{\mathrm{a}^{\prime}}{\mathrm{c}^{2}{ }^{2}} \mathrm{c}\right)
$$

Pour expliquer cette relation, il est vraisemblablement nécessaire de fairc intervenir plusieurs réactions. On considère les deux réactions qui correspondent aux deux critères qui ont guidé cette étude :

- structure diviséc, réaction de surface, sites actifs $(\sigma)$;
- catalyseur d'oxydation : oxydation et régénération des sites actifs.

Ces réactions peuvent s'écrire :

$$
\mathrm{Mn}^{++}+\sigma \underset{\mathrm{K}_{2}}{\stackrel{\mathrm{K}_{1}}{\rightarrow}} \mathrm{Mn} \sigma^{++}
$$

$\mathrm{Mn} \sigma^{++}+\mathrm{H}_{2} \mathrm{O}+\frac{\mathrm{x}-1}{2} \mathrm{O}_{2} \stackrel{\mathrm{K}_{3}}{\longrightarrow} \mathrm{MnOx}(\sigma)+2 \mathrm{H}^{+}$

Dans la deuxième réaction, $\mathrm{MnOx}(\sigma)$ représente l'oxyde formé constituant un site actif traduisant le phénomène de catalyse. Une étude mathématique de ce modèle montre que la vitessc de démanganisation peut s'écrire de la façon suivante :

$$
-\frac{\mathrm{dc}}{\mathrm{dt}}=\frac{\mathrm{K}_{3} \mathrm{ScP}\left(\mathrm{O}_{2}\right)\left\lfloor 1+\frac{\mathrm{K}_{2}}{\mathrm{~K}_{1}} \mathrm{c}\right]}{\left.1+\frac{\mathrm{K}_{2}}{\mathrm{~K}_{1}} \mathrm{c}\right]^{2}}
$$

Ge modèle correspond à un équilibre d'absorption instantané. Si l'on admet une hypothèse d'état stationnaire pour les composés intermédiaires $\mathrm{Mn} \sigma$ et $\mathrm{MnOx}(\sigma)$, on peut obtenir une relation du type suivant :

$$
-\frac{\mathrm{dc}}{\mathrm{dt}}=\frac{\mathrm{K}_{2} \cdot \mathrm{K}_{3}}{\mathrm{~K}_{2}+\mathrm{K}_{3}} \mathrm{c}\left[\left(\sigma_{0}\right)-\mathrm{c}_{\mathrm{o}}+\mathrm{c}\right]
$$

Ce type de relation du sccond degré en c est assez proche de la loi empirique trouvée $\sigma_{0}$ et $c_{0}$ sont respectivement le nombre de sites actifs initiaux et la concentration initiale. Elle permet de rendre compte que suivant les valeurs respectives de $c_{o}$ et $c_{o}$, la cinćtique globale de la réaction peut avoir un ordre apparent variant cntre 1 et 2 .

\section{POSSIgILITÉ D'Élimination D'AUTRES CATIONS}

Des expériences analogues à celles qui ont été réalisées pour l'étude de la démanganisation ont été effectuées vis-à-vis de $\mathrm{Cu}, \mathrm{Ni}, \mathrm{Zn}$. $\mathrm{Hg}$, $\mathrm{Pb}$. La figure 8 présente les résultats obtenus sur différents types d'oxydes de manganèse. Dans le cas du cuivre comme dans le cas du manganèse, la supériorité du matériau " nodule » est évidente : il n'y a pas d'efficacité très dif- férente entre les nodules pacifique et atlantique (Walda).

La figure 9 montre clairement une efficacité des nodules différente suivant les différents métaux considérés : les éléments peuvent être classés de la façon suivante en fonction d'une efficacité décroissante : $\mathrm{Mn}, \mathrm{Cu}, \mathrm{Ni}, \mathrm{Zn}, \mathrm{Hg}$, $\mathrm{Pb}$. 


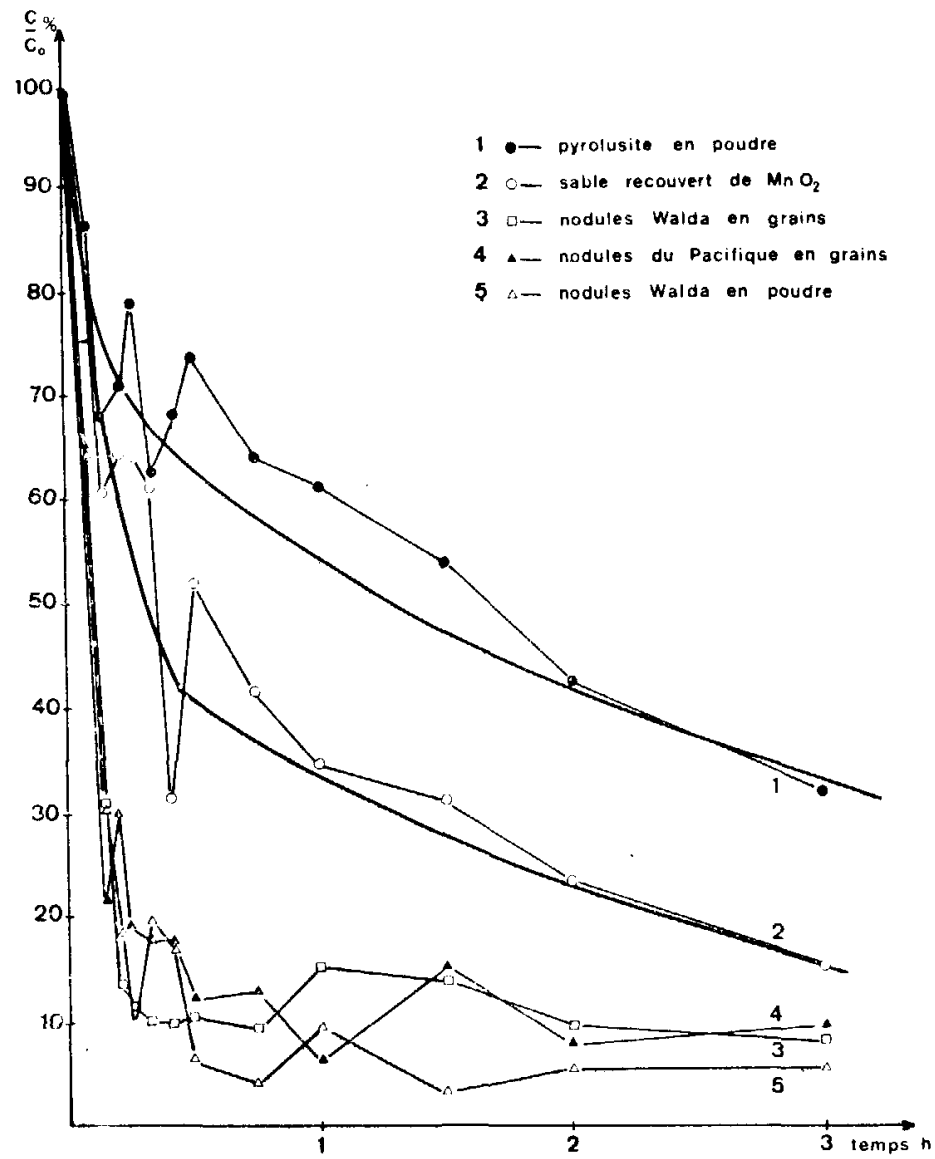

Fig. 8 Elimination du cuivre sur differents types doxydes de manganèse

Cu removal using different manganese oxyde types : 1 - pyrolusite (powder); 2 - manganese coated sand; 3 - nodules "Walda" (sand size); 4 -nodules from Pacific Ocean (sand size); 5 -nodules "Walda" (powder).

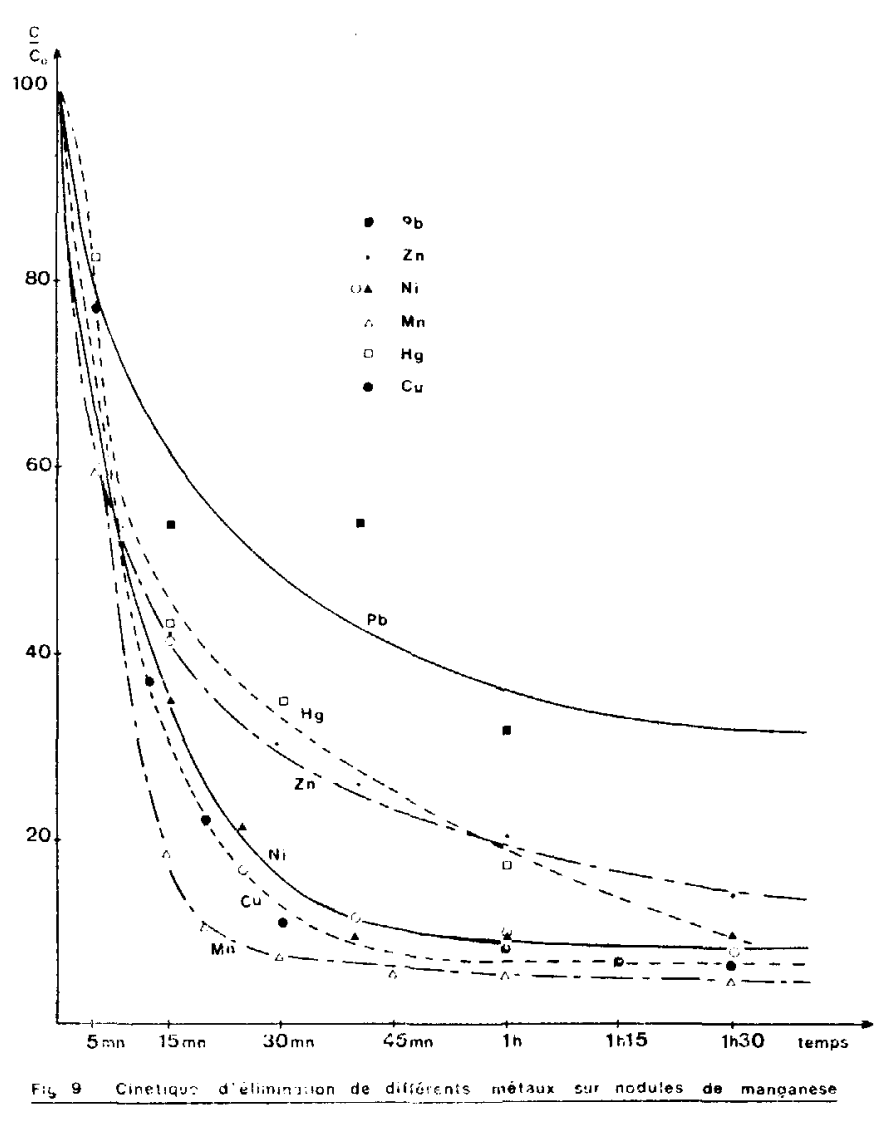

FIGURE: 9

Efficacité des nodules vis-à-vis de différents matériaux. Compared efficiency of nodules for differend elements.

\section{EFFICACITÉ DES DIFFÉRENTS DISPOSITIFS EXPÉRIMENTAUX}

Les différentes expériences mentionnées jusqu'ici ont pour la plupart été effectuées en discontinu. Elles ont permis de tester le matériau " nodule de manganèse " par comparaison avec d'autres matériaux plus classiques, d'étudier la cinétique de la réaction de démanganisation d'une solution et enfin, observation très importante, de montrer que le matériau pouvait se régénérer.

Envisageant d'utiliser les nodules de manganèse comme agent de démanganisation des eaux, trois montages ont été testés du point de vue de leur efficacité.

I - Une colonne descendante (hauteur $25 \mathrm{~cm}$, diamètre $3 \mathrm{~cm}$ ), l'ćcoulement de la solution se faisant par gravité à débit constant (fig. 10).

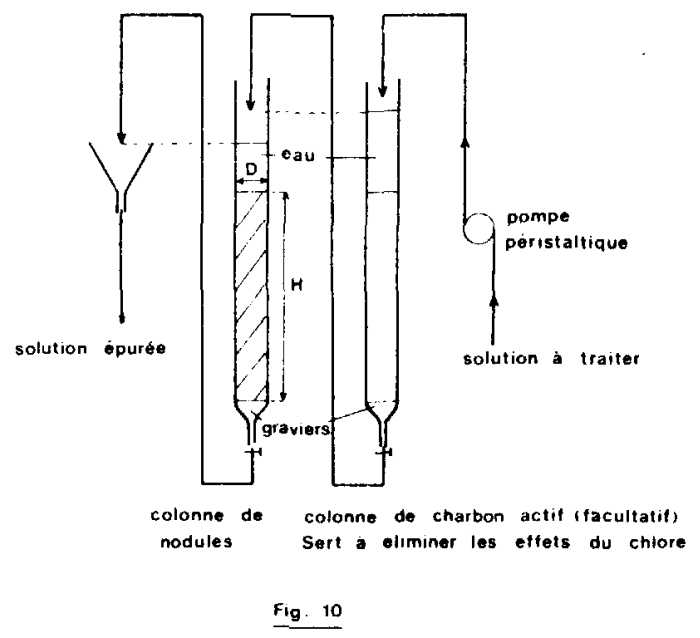

Figure, 10

Colonne descendante.

Descending column. 
II - Une colonne ascendante, le lit de nodules étant mis en expansion suivant le principe du courant (fig. 11).

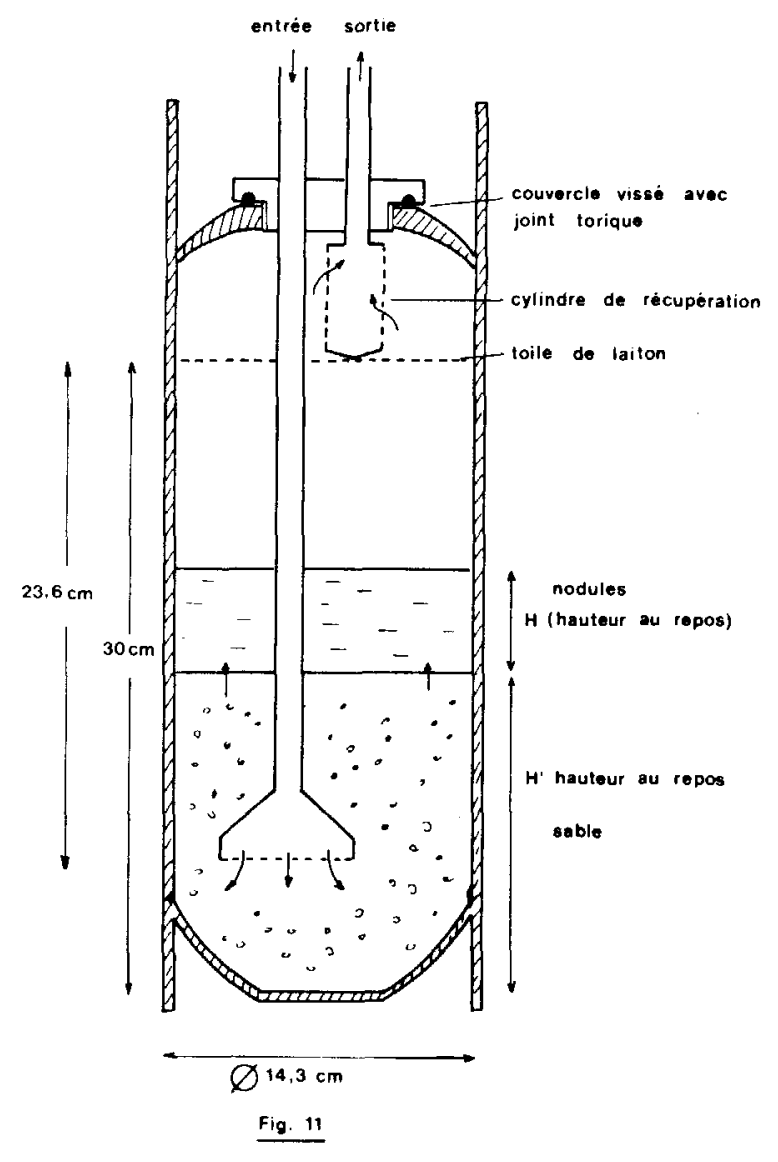

FIGURE 11

Colonne ascendante.

Ascending column.

III - Un lit horizontal (fig. 12), longueur $55 \mathrm{~cm}$, hauteur $5 \mathrm{~cm}$, l'eau s'écoulant par gravité dans un bac légèrement incliné, les nodules affleurant à la surface de l'eau.

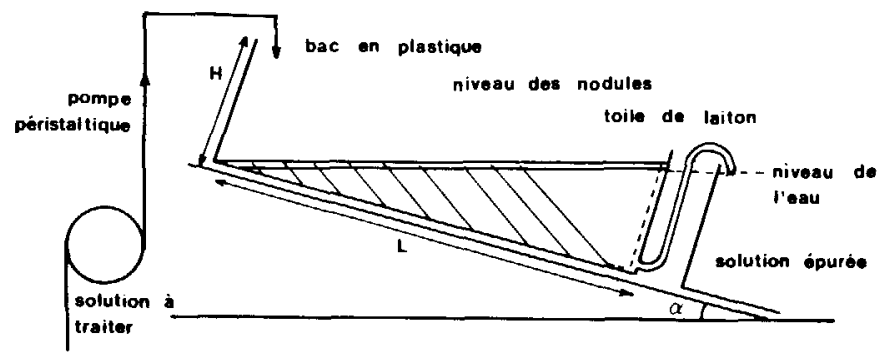

Fig. 12

FIGURE 12

Lit horizontal.

Horizontal bed.
Pour caractériser chaque expérience effectuée avec l'un ou l'autre de ces montages, deux critères ont été choisis : $\mathrm{T}$, temps au-delà duquel la concentration en $\mathrm{Mn}^{++}$à la sortie dépasse $0,1 \mathrm{mg} / \mathrm{l}$, et $\mathrm{V}$, «vitesse de saturation », pente moyenne de $\mathrm{c}=\mathrm{f}(\mathrm{t})$ à la sortie de l'installation. Les résultats sont présentés en tableau III ; figurent aussi dans ce tableau les résultats obtenus pour $\mathrm{Cu}$ et $\mathrm{Zn}$ (pour $\mathrm{T}$, les concentrations choisies sont 0,5 et $0,7 \mathrm{mg} / 1$ respectivement).

Pour les montages I ct II on peut supposer que le temps $\mathrm{T}$ serait prolongé avec une couche plus épaisse de nodules. La comparaison entre ces deux systèmes est délicate du fait de la différence dans leur dimensionnement. On peut toutefois noter que $\mathrm{V}$ semble plus faible à conditions équivalentes, dans le montage II : la surface de contact entre solide et liquide serait meilleure dans ce type de montage. Il faut remarquer que les conditions sont très différentes, et souvent moins bonnes, en laboratoire que dans les grandes unités (effets de paroi par exemple) et que les conclusions devront être vérifiées sur des pilotes à une échelle plus importante.

Le $\mathrm{pH}$ d'entrée a peu d'influence. On peut cependant noter une petite amélioration à $\mathrm{pH}$ légèrement basique $(8,5)$ qui favorise la précipitation des hydroxydes. La concentration d'entrée est un facteur de grande importancc. V la vitesse de saturation n'est pas proportionnclle à la concentration d'entrée mais croît beaucoup plus vite que $c_{0}$ croît. C'est un résultat favorable à une éventuclle application à des eaux naturelles dont les teneurs en métaux restent toujours faibles. Le débit, et, par suite, la charge hydraulique ont une très grande influence sur $\mathrm{V}$ : plus la charge hydraulique est grande, plus la colonne se sature vite.

Les différents métaux ne sont pas éliminés de façon équivalente par les nodules : la comparaison entre $\mathrm{Cu}^{2+}$ et $\mathrm{Zn}^{2+}$ dans la même expéricnce montre que la saturation des nodules vis-à-vis du zinc est plus rapide, comme l'ont laissé prévoir les essais préalables en discontinu.

La comparaison des résultats suivant les trois montages montre une supćriorité évidente du montage «lit horizontal»; ce montage favorise l'aération. 
TABLEAU III

Résultats expérimentaux relatifs aux différents montages : colonne descendante, colonne ascendante, lit hotizontal.

\begin{tabular}{|c|c|c|c|c|c|c|}
\hline $\begin{array}{l}\text { Type de montage } \\
\text { sel introduit }\end{array}$ & $\mathrm{pH}$ & $\begin{array}{l}\text { Charge volumique } \\
\qquad \mathrm{m}^{3} / \mathrm{h} / \mathrm{m}^{3}\end{array}$ & $\begin{array}{l}\text { Charge hydraulique } \\
\mathrm{m} \mathrm{m}^{3} / \mathrm{h} / \mathrm{m}^{3}\end{array}$ & $\begin{array}{l}\text { Concentration } \\
\text { d'entrée } C_{0} \\
\text { mg/l de métal }\end{array}$ & $T$ & $\underset{\mathrm{mg} / 1 / \mathrm{h}}{\mathrm{V}}$ \\
\hline $\begin{array}{l}\text { I colonne } \\
\text { descendant }\end{array}$ & 7 & & 2,5 & 5 & 2,5 & \\
\hline & 8,2 & & 2,5 & 8,6 & 4,5 & \\
\hline$\left[\mathrm{MnSO}_{4}\right]$ & $\begin{array}{l}7-7,5 \\
7-7,5\end{array}$ & - & $\begin{array}{l}4 \\
2,5 \\
2,8 \\
5,3 \\
1,4 \\
1,9 \\
2,8\end{array}$ & $\begin{array}{r}1 \\
5 \\
30 \\
100 \\
45 \\
45 \\
31\end{array}$ & $\begin{array}{l}30 \\
2,5 \\
1\end{array}$ & $\begin{array}{c}0,0 \\
0,03 \\
4,4 \\
26,0 \\
0,5 \\
1 \\
3\end{array}$ \\
\hline $\begin{array}{l}\text { II colonne } \\
\text { ascendant } \\
{\left[\mathrm{MnSO}_{4}\right]}\end{array}$ & & $\begin{array}{l}6,2 \\
6,2\end{array}$ & $\begin{array}{l}0,3 \\
0,6\end{array}$ & $\begin{array}{l}2 \\
2\end{array}$ & 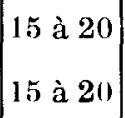 & $\begin{array}{l}0,310^{-2} \\
0,710^{-2}\end{array}$ \\
\hline $\begin{array}{l}\text { III lit horizontal } \\
{\left[\mathrm{MnSO}_{4}\right]}\end{array}$ & & $\begin{array}{l}0,85 \\
2\end{array}$ & & $\begin{array}{l}13 \\
14\end{array}$ & $\begin{array}{l}360 \\
180\end{array}$ & \\
\hline$\left[\mathrm{CuSO}_{4}\right]$ & 4 à 6 & 2 & & 20 & 130 & \\
\hline $\begin{array}{l}{\left[\mathrm{CuSO}_{4}\right]} \\
{\left[\mathrm{Zn} \mathrm{Cl} \mathrm{Cl}_{2}\right]}\end{array}$ & 4 à 6 & 4 & & $\begin{array}{l}C u=1,5 \\
Z n=2\end{array}$ & $\begin{array}{l}1200 \\
720\end{array}$ & \\
\hline
\end{tabular}

\section{CONGLUSION}

Deux hypothèses ont servi de base à une utilisation possible des nodules de manganèse pour l'élimination de certains éléments présents dans les eaux ( $\mathrm{Mn}, \mathrm{Cu}, \mathrm{Zn}, \mathrm{Hg}, \mathrm{Pb})$ :

- le dioxyde de manganèse possède des propriétés catalytiques vis-à-vis de certaines réactions d'oxydation ( $\mathrm{Mn}^{++}$notamment) ;

- le matériau possède une structure poreuse favorable aux réactions de surface.

Ces deux hypothèses se trouvent largement vérifiées par les séries d'expériences réalisées :

- le matériau "nodule de manganèse" est plus efficace que les matériaux classiques pyrolusite ou sable manganisé ;

- dans l'ordre décroissant l'efficacité d'élimination des éléments est la suivante : $\mathbf{M n}$, $\mathrm{Cu}, \mathrm{Ni}, \mathrm{Zn}, \mathrm{Hg}, \mathrm{Pb}$;

- le matériau étant "saturé", il peut se régénérer de lui-même ;
- la saturation ou la régénération du matériau est la conséquence de la cinétique globale des dcux réactions suivantes :

$$
\begin{gathered}
\mathrm{Mn}^{2}+\sigma \rightleftarrows \mathrm{Mn} \sigma^{2^{+}} \\
\mathrm{Mn}^{2+}+3 \mathrm{H}_{2} \mathrm{O}+\frac{\mathrm{x}-\mathrm{l}}{2} \mathrm{O}_{2} \rightarrow \mathrm{MnO}_{\mathrm{x}}, \sigma+2 \mathrm{H}_{3} \mathrm{O}^{+}
\end{gathered}
$$

- le pH n'a pas ou peu d'effet sur la cinćtique (réaction irréversible);

- parmi les trois types de montage «en continu " testés (colonne descendante, colonne ascendante et lit horizontal), le dispositif lit horizontal est le plus satisfaisant, permettant la meilleure oxygénation du milieu.

Cette étude a été entreprise dans l'esprit d'une diversification possible de l'emploi des nodules de manganèse, ce matériau faisant 
actucllement l'objet d'investigations en tant que minerai potentiel de cuivre et de nickel. Bien que les expériences aient été effectuées en milieu eau douce, il convient de noter que quelques informations peuvent être déduites vis-à-vis de la formation des nodules :

- pourvu que de l'oxygène soit disponible, la croissance des nodules peut s'entretenir simplement par réaction chimique - sans qu'il soit nécessaire de faire intervenir un aspect biologique ;

- les nodules possèdent un très bon pouvoir d'absorption du cuivre et du nickel (nettement meilleur que pour les autres ćléments étudiés,
$\mathrm{Zn}, \mathrm{Hg}$ ou $\mathrm{Pb})$; cette observation est à rapprocher de la corrélation des teneurs $\mathrm{Mn}$, Cu, $\mathrm{Ni}$ qui existe dans les nodules;

- les nodules atlantiques "Walda" très riches en manganèse font exccption à la corrélation $\mathrm{Mn}, \mathrm{Cu}, \mathrm{Ni}$ : leurs concentrations en $\mathrm{Ni}$ et $\mathrm{Cu}$ sont très faibles. Leur pouvoir d'absorption du $\mathrm{Cu}$ et $\mathrm{Ni}$ est aussi élevé que celui des nodules du Pacifique. Les très faibles tencurs en $\mathrm{Ni}$ ct $\mathrm{Cu}$ ne sont pas liées à une proprićté particulière de ces nodules. Les nodules "Walda " ont été prélevés en bas de marge africaine ; la source des éléments formant ces nodules est donc très certainement de nature différente de celle qui forme les nodules du Pacifique.

\section{RÉFÉRENCES}

Coughis R.W., Matsui I. - Catalytic oxydation of aqueous MnII. Journ. Catal. (1976), 41, p. 108-123.

Gr,asBy, éditeur. Marine Manganese deposits. Hisevier, Oceanography series, 15 (1977).

Pignetit G. $(197 x)$. - Thèse de Doctenr, Ingénieur, Université de Rennes, Icole Nationale Supérieure de Chimie de Rennes.

MORGAN JJ., STUMm W. - Analytical Chemistry of aqueous manganese. JAWWA (1977), 57, p. 107119.

NoRDELIE, E. -- Iron and Manganèse removal water sewage works (mai 195:3), p. 18L-185.

\section{RHMERCILMFNTS}

Ce travail a été réalisé dans le cadre d'un contrat d'étude du Centre National pour l'Iixploitation des Océans.

\section{Résumé}

I,es nodules polymétalliques riches en bioxyde de manganèse sont utilisés afin d'enlever des eaux des traces de micropolluants (cations métalliques). I'étude théorique cinétique et les mécanismes des réactions sont discutés dans le cadre de la démanganisation. L'efficacité des nodules est supérieute à celle des autres méthodes de déman1ganisation. Ia technologie de mise en auvre d'unités pilotes travaillant en continu est discutée.

\section{Abstract}

Manganese rich polymetallic nodules are used for trace element removal from fresh-water. The theory of kinetics and mechanisms of reactions are discussed for manganese removal. The efficiency of nodules is higher than efficiencies related to other manganese removal methods. Pilot units involving continuous processes are discussed.

Polymetallic nodules and polluted fresh-water treatment. 